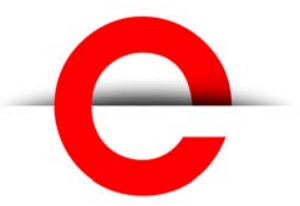

U T S

e PRES S
PORTAL Journal of Multidisciplinary International Studies

Vol. 17, No. 1/2

Jan 2021

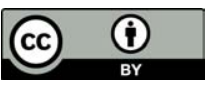

(C) 2021 by the author(s). This is an Open Access article distributed under the terms of the Creative Commons Attribution 4.0 International (CC BY 4.0) License (https:// creativecommons.org/ licenses/by/4.0/), allowing third parties to copy and redistribute the material in any medium or format and to remix, transform, and build upon the material for any purpose, even commercially, provided the original work is properly cited and states its license.

Citation: Hassan, C., and Mangiapane, G. 2021.

Respirare il tempo:

Re-Azioni di superamento COVID-19. PORTAL Journal of Multidisciplinary International Studies, 17:1/2, 36-44. http:// dx.doi.org/10.5130/pjmis. v17i1-2.7339

ISSN 1449-2490 | Published by UTS ePRESS | http://epress. lib.uts.edu.au/ojs/index.php/ portal

\title{
ESSAY
}

\section{Respirare il tempo: Re-Azioni di superamento COVID-19}

\section{Cadigia Hassan and Gianluigi Mangiapane}

Corresponding author(s): Cadigia Hassan, freelance journalist. Padua, Italy. cadigiahassand hotmail.com

Gianluigi Mangiapane, Department of Philosophy and Educational Sciences, University of Turin. Corso Massimo d’Azeglio, 52 I-10126 Turin, Italy. gianluigi.mangiapaneßunito.it

DOI: http://dx.doi.org/10.5130/pjmis.v17i1-2.7339

Article History: Received 20/07/2020; Accepted 11/11/2020; Published 28/01/2021

\begin{abstract}
This reflective essays retraces the experience of 'Mirabilia', an online project originated at the beginning of the Italian lockdown (March 2020) by a spontaneous group made up of researchers from different disciplines, which had the purpose of showing human creativity and the ability to find strategies for survival and ways to cope with adversity. In particular, the essay illustrates "The Breath of Time", a virtual exhibition created by Mirabilia: through a public call, many images and narrations of personal and particularly significant objects were collected during the quarantine, and are here discussed and illustrated.
\end{abstract}

\section{Keywords}

\#iorestoacasa; Iniziative Dal Basso; Mostra Virtuale

\section{Premessa ${ }^{1}$}

Il lungo periodo di lockdown che l'Italia si è trovata ad affrontare, dal 9 marzo al 3 giugno 2020, ha portato in tutti noi un senso di smarrimento, paura e incertezza. Sia come singoli cittadini che come istituzioni ci siamo trovati impreparati ad affrontare un nemico invisibile

1 Acknowledgement: L'autrice e l'autore di questo saggio confermano che parti dello stesso compaiono nel blog 'Il respiro del tempo,' in particolare alla pagina https://www.respirodeltempo.it/mostra-e-percorsiespositivi/. [The authors acknowledge that parts of this essay appear on the blog 'Il respiro del tempo,' on the page https://www.respirodeltempo.it/mostra-e-percorsi-espositivi/]. 
e subdolo, e per questo ancor più pericoloso. Una cosa che è subito emersa è stata l'assoluta democraticità del COVID-19: un virus che colpisce indiscriminatamente, livellando le differenze sociali, di genere, economiche, abitative, di provenienza. La crisi pandemica ha tuttavia messo in risalto vari gap tra la cittadinanza: tra chi ha un'abitazione in cui ha potuto osservare quel martellante quanto indispensabile monito 'Restate a casa' (tradotto per la comunicazione via social nell'hashtag \#iorestoacasa) e chi invece è stato costretto a vivere per strada o in contesti di sovraffollamento (si pensi, ad esempio, ai senza fissa dimora, ai richiedenti asilo ospitati nei centri di accoglienza o agli anziani accolti nelle RSA); tra chi, studente di ogni ordine e grado, poteva contare su una $\mathrm{DaD}$ (Didattica a Distanza) fluida e chi invece è stato rallentato dalla mancanza di dispositivi elettronici o da difficoltà di connessione; tra chi ha potuto contare sullo smart working e chi invece ha perduto o sospeso il lavoro. Si pensi, per esempio, ai precari, ai numerosi lavoratori a chiamata o ai prestatori sommersi di manodopera lavorativa (fenomeno piuttosto diffuso in Italia). Il COVID-19 è stato quindi un disequalizzatore dalle dimensioni variabili quanto soggettive.

Per quanto riguarda le differenze in materia di accessi e consumi culturali, possiamo però dire che vi è stata una frenetica ed entusiasmante corsa livellatrice, sia top down (dalle istituzioni) che bottom up (dal basso). La crisi e l'esperienza di vivere in isolamento si sono intersecate con la creazione artistica e la conoscenza e potremmo citare un'infinità di esempi open access, in cui la cultura è venuta incontro al singolo, permettendogli fruizioni e interazioni altrimenti inaccessibili, facendolo sentire partecipe, protagonista, come in prima fila, nonostante la distanza mediata, e pertanto meno solo, isolato, angosciato e più stimolato e catapultato in un virtuale acculturato e incantato. Il Ministero della Cultura italiano ha da poco presentato un report relativo a uno studio realizzato dal Politecnico di Milano su cento luoghi statali della cultura e i risultati sono molto significativi in quanto 'durante l'emergenza pandemica, le attività di musei, parchi archeologici e istituti si sono riversate sui social e questo ha contribuito a raddoppiare la loro visibilità sul web, e di conseguenza la reputazione online, rispetto al periodo pre-Covid' (Giraud 2020: paragrafo 1). Questo ha portato molti musei a un continuo dialogo con il proprio pubblico e a una maggiore accessibilità all'offerta culturale rispetto al periodo precedente.

\section{Iniziative dal basso: il caso di 'Mirabilia'}

Non solo le istituzioni statali e private sono state molto attive nel web in questi mesi, ma anche i singoli cittadini o gruppi che si sono formati in maniera spontanea hanno collaborato al benessere della comunità e contribuito al welfare culturale del Paese, dove per 'welfare culturale' intendiamo la collocazione di

processi di produzione e disseminazione culturale all'interno di un sistema di welfare, (...) che garantiscono ai cittadini le forme di cura e accompagnamento necessarie al superamento di criticità legate alla salute, all'invecchiamento, alle disabilità, all'integrazione sociale e a tutte le problematiche a cui si associa il riconoscimento di un dovere di tutela sociale. (Sacco 2017: paragrafo 1)

Le iniziative culturali lanciate dal basso, da singole persone o da gruppi, in tempo di coronavirus sono state numerose in Italia e sarebbe impossibile citarle tutte: hanno rappresentato comunque una risposta emotiva al vivere l'isolamento e talvolta sono andate a sostituire relazioni sociali in un lungo periodo con contatti fortemente limitati. Fra queste però desideriamo segnalare il progetto 'Mirabilia': in latino, il termine mirabilia indica 'meraviglie,' oggetti o circostanze che provocano uno stato spontaneo di ammirazione e stupore, un compiacimento reso ancor più esaltante per l'eccezionalità e l'imprevedibilità della sua manifestazione. In realtà, in latino, ha anche un'altra accezione, indirizzata alla letteratura periegetica e di viaggio: mirabilia erano delle guide create allo scopo di orientare pellegrini e viaggiatori nel loro peregrinare. Questa iniziativa è stata lanciata all'alba del contenimento in Italia, proprio il 9 marzo scorso, 
da un gruppo, in continuo divenire, formato da persone impegnate in diversi ambiti culturali. ${ }^{2}$ Lidea alla base di questo progetto consiste nel pubblicare quotidianamente una o più mirabilia, ovvero un oggetto, un evento o una pratica proveniente da diverse culture del mondo (sia vicine che lontane, nel tempo e nello spazio). L'obiettivo è quello di sorprendere, affascinare e meravigliare i lettori e le lettrici, mostrando la creatività dell'essere umano, anche nell'applicare strategie di sopravvivenza, modi di fronteggiare le avversità e il perturbante. Le immagini e i corrispettivi articoli hanno trovato ospitalità sia sul blog, denominato 'CulturaeMirabilia,' sia sulla pagina Facebook del gruppo. ${ }^{3}$

\section{Il respiro del tempo}

Tra i vari lanci di Mirabilia c'è poi stata anche una call pubblica promossa per raccogliere immagini e narrazioni dei personali oggetti d'affezione durante la quarantena. L'iniziativa ha riscontrato un buon gradimento e ha dato vita a un progetto autonomo: la mostra virtuale 'Il respiro del tempo,' è stata visitabile online fino al 15 settembre, in italiano e inglese. ${ }^{4}$

La mostra parte dal presupposto che gli oggetti non sono mai neutri (Miller 1991; Miller 2005), ma sono simbolici di un periodo, un affetto, un desiderio, un malessere o una memoria, e sono al centro di reti di relazione che ci aiutano a conoscere la realtà e noi stessi. Il percorso, quindi, è costituito dalle immagini di una trentina di oggetti scelti dalle persone che hanno voluto rispondere alla chiamata. I e le partecipanti hanno quindi mandato le fotografie di quegli oggetti—corredate da narrazioni personali-che hanno ritenuto significativi durante la pandemia 2020. L'idea alla base di questo progetto antropologico non era tanto quella di una rappresentazione corale tout court, quanto di lasciare — attraverso la rete—una testimonianza che consenta di ricostruire e visualizzare, nei tempi a venire, il 'sentire' collettivo di un periodo di lockdown, timore e incertezza.

Alla mostra virtuale hanno contribuito una trentina di autori e autrici, provenienti da Italia, Canada, Grecia, Lituania, Spagna e Svezia, di diverse età e professioni (e magari altri ancora contribuiranno in seguito, in quanto la call resta aperta fino alla chiusura della mostra). A proposito degli oggetti presenti in mostra, Alfredo Sgarlato, uno dei curatori, scrive sul sito web dell'iniziativa:

Molti degli oggetti scelti per questa esposizione virtuale manifestano un desiderio: il significato etimologico della parola 'desiderio' rimanda al prigioniero, che sente la mancanza del cielo stellato, quindi della libertà. Così, tra gli oggetti fotografati dai partecipanti alla nostra chiamata, abbiamo molte scelte che si rifanno, inevitabilmente, al desiderio in senso più stretto, un moto dinamico verso l'altrove, che sia pratico (le scarpe, la cartina), o immaginario (il pennello, il taccuino). ( 2020: paragrafo 1)

In alcuni casi, poi, gli oggetti rappresentano una soglia tra il dentro e il fuori, come, per esempio porte o sbarre, tra il passato e il futuro, facendo emergere un altro tipo di desiderio (ig. 1 ):

la volontà 'di una continuità temporale' dove 'il prima (della quarantena) non rimanga un passato isolato ma possa connettersi al futuro, con gli oggetti come testimonianza di un passaggio a una nuova fase. (paragrafo 1).

2 L'autrice e l'autore del contributo fanno parte del gruppo di 'Mirabilia', nato in maniera del tutto spontanea.

3 Per il sito web si veda: https://culturaemirabilia.blogspot.com/ (2020) [Accessed 16 November 2020]; mentre per la pagina Facebook si veda sul social network: Aculturaemirabilia.

4 Si veda il sito web: www.respirodeltempo.it (2020) [Accessed 16 November 2020]. 
Secondo il curatore: 'non manca la funzione apotropaica (degli oggetti, n.d.a.), inevitabile in un momento in cui il futuro è un'incognita' (paragrafo 1).

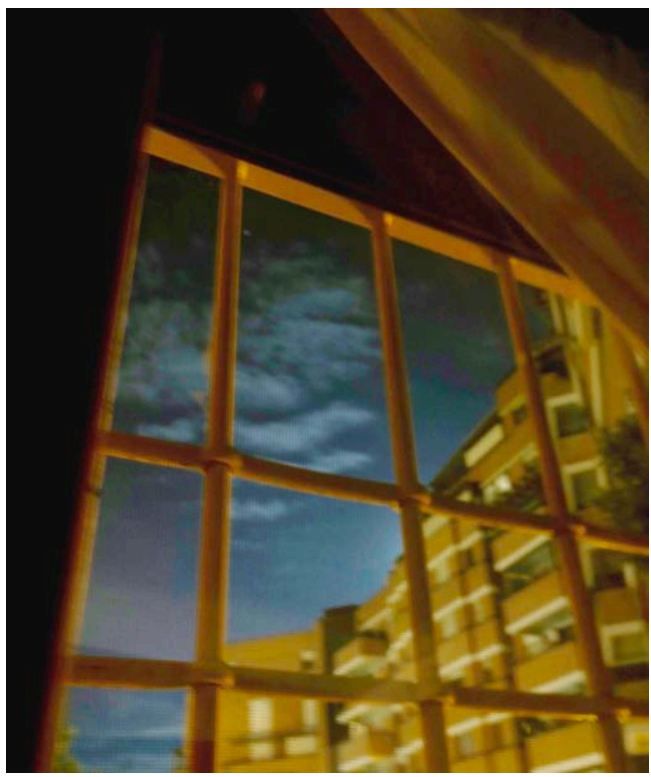

Figure 1. Sbarre alla finestra, 2020; ๔ Claudia Marchi

Inoltre, lo stesso autore sostiene che 'osservare questa serie di oggetti rinforza una riflessione,' ovvero che il lockdown 'ha riportato l'attenzione su un argomento che appariva tabù nella società attuale, dominata dal culto del profitto e dal principio di prestazione (il "discorso del capitalista," direbbe Lacan): il diritto al tempo libero, come elemento fondante dell'identità personale e collettiva' (Sgarlato 2020: paragrafo 3). Del resto, questi 'oggetti quotidiani diventano parti indissolubili di noi, così come parti di una totalità assoluta' (paragrafo 4).

La prima tentazione di Mirabilia è stata quella di catalogare gli oggetti d'affezione pervenuti in aree tematiche, ma catalogare significa rinchiudere in gabbie interpretative fisse e relativistiche, quando invece ogni oggetto è in sé polisemico, evoca cioè immagini e significati soggettivi a seconda del vissuto personale e del contesto culturale e sociale di appartenenza (Hassan 2020: paragrafo 1). Si è deciso così di concentrarsi invece sul legame emozionale con l'oggetto, divenuto per alcuni transizionale per la sua capacità di offrire riparo, protezione e conforto psicologico.

Oggetti che sottolineano un 'dentro'e un 'fuori,' un 'prima' e un nunc sospeso. Le sbarre alla finestra che un tempo tenevano al sicuro da ogni intrusione ora si fanno pesanti perché riflettono l'idea di essere in trappola pur se allo stesso tempo consentono di vedere comè il mondo 'dall'altra parte.' Le pantofole di lana e gli zoccoli estivi che dialogano attraverso una porta a vetri (fig.2) rimandano a un tempo che verrà, ciclico e tenace come il tempo della natura, racchiuso in una piantina di basilico vittoriosamente sopravvissuta all'inverno (paragrafo 1). 
U T S

e PRES S

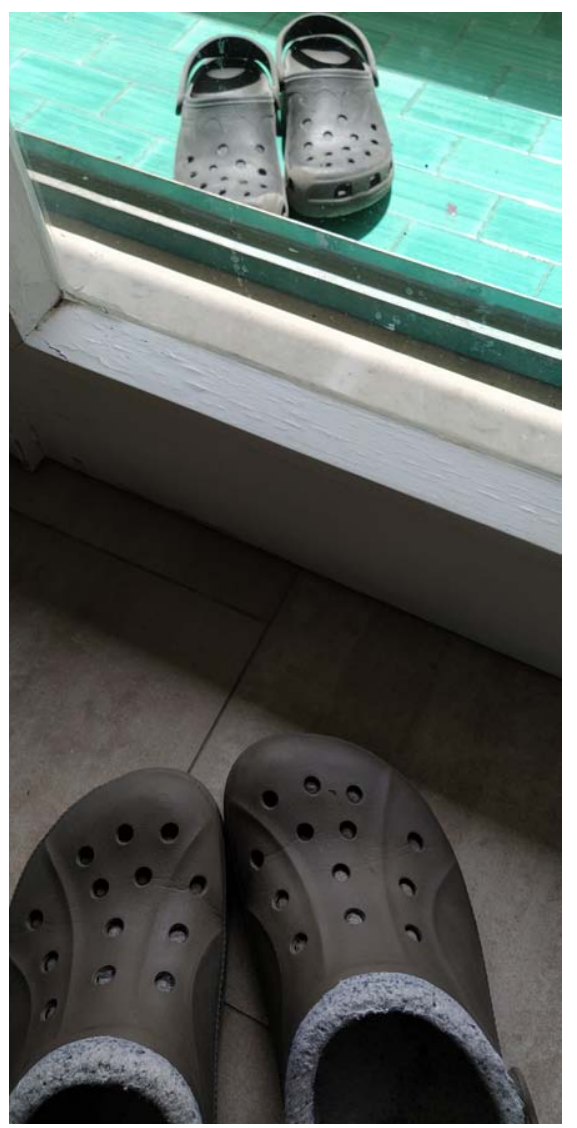

Figure 2. Pantofole di lana e zoccoli estivi in dialogo attraverso una porta, 2020; (c) Mariella Brenna.

L'immagine di una soglia, oggettiva o simbolica, di un limen, è molto ricorrente in questi contributi. D’altronde la stessa pandemia può considerarsi un rito di passaggio con i suoi tre stadi di separazione, transizione e reintegrazione (Van Gennep 1909). Le scarpe lasciate sullo zerbino, senza testo descrittivo, spingono la mente a trovare molteplici allusioni mentre la porta a vetri di un corridoio di casa, che impedisce a un marito affetto da corona virus di contagiare la propria moglie, testimonia l'idea di confinamento nel confinamento, di un'assenza in co-presenza, con tutto il portato di solitudine, paure, pensieri e strategie di sopravvivenza (Hassan 2020: paragrafo 2).

Perché è questo il primo pensiero dettato dall'istinto di conservazione: sopravvivere, ovvero 'vivere sopra,' al di là del rischio e del pericolo, per continuare ad esistere, ad esserci, a farsi testimoni. A questo pensiero ci si può aggrappare con la fantasia, con la creatività o con un oggetto dalle virtù apotropaiche: le famiglie ritagliate su carta che prendono forma per 'andare restando'; l'albero secolare in giardino con la sua ferita di guerra, simbolo di resilienza (fig. 3 ); il restauro del tavolo della nonna, custode di immagini e memorie lontane; un braccialetto-amuleto, un rosario, delle pietre significative o una mattonella in tessuto per allontanare lo stress e ritrovare la pace dell'anima; una poesia sull'immagine di un'alba o un taccuino per fissare le sensazioni e le tappe di questo tempo straordinario. 
U T S

e PRESS

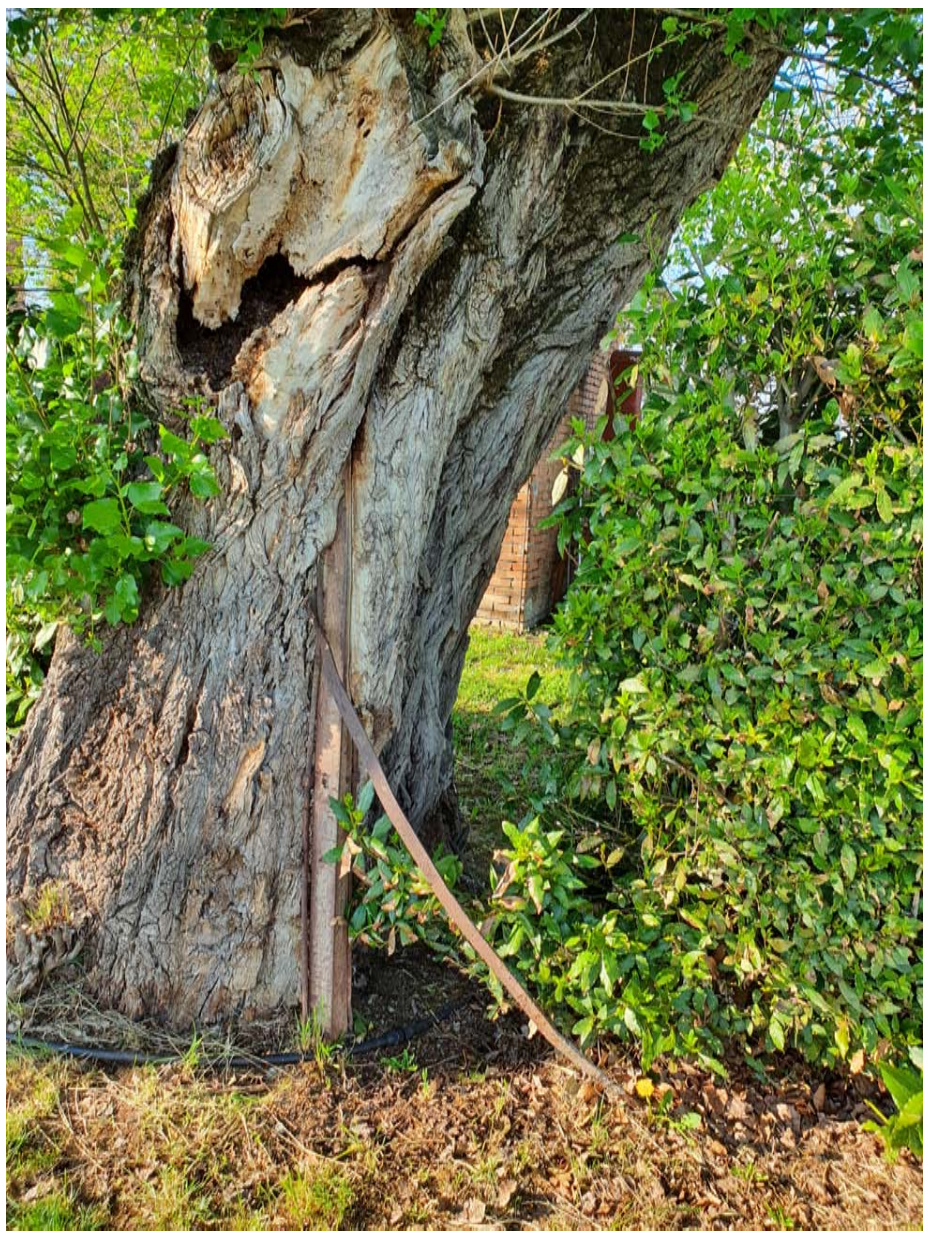

Figure 3. Albero secolare con la ferita di guerra, 2020; @ Federica Santoro

Un tempo costellato da diverse scansioni, che diventa pausa, attesa, silenzio, riflessione, occasione per staccarsi dal mondo, liberarsi dalle gabbie mentali e abbracciare il vero sé. Un tempo 'umanizzato,' perduto e ritrovato in un autoritratto, in un'incitazione scritta sulla propria tazza preferita o attraverso il distacco regalato da un paio di cuffie auricolari (fig.4). E poi c'è l'evasione, l'arte del pensiero di fuggire da uno spazio che ci reclude e angoscia, rifugiandosi nei libri, inventandosi nuovi orizzonti attraverso pennelli e tavolozza (fig.5), perdendosi nell'infinita quiete del mare visto attraverso la finestra o promettendosi, davanti a una cartina geografica, di ritornare presto a vivere persone e luoghi conosciuti o inesplorati (paragrafo 2). 


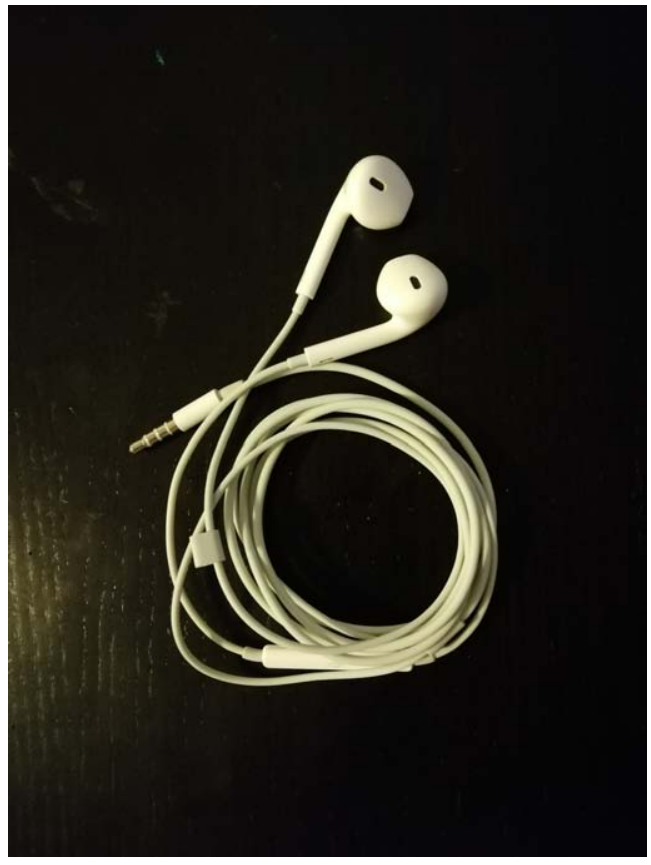

Figure 4. Auricolari per smartphone, 2020; @ Gabriela Diaz

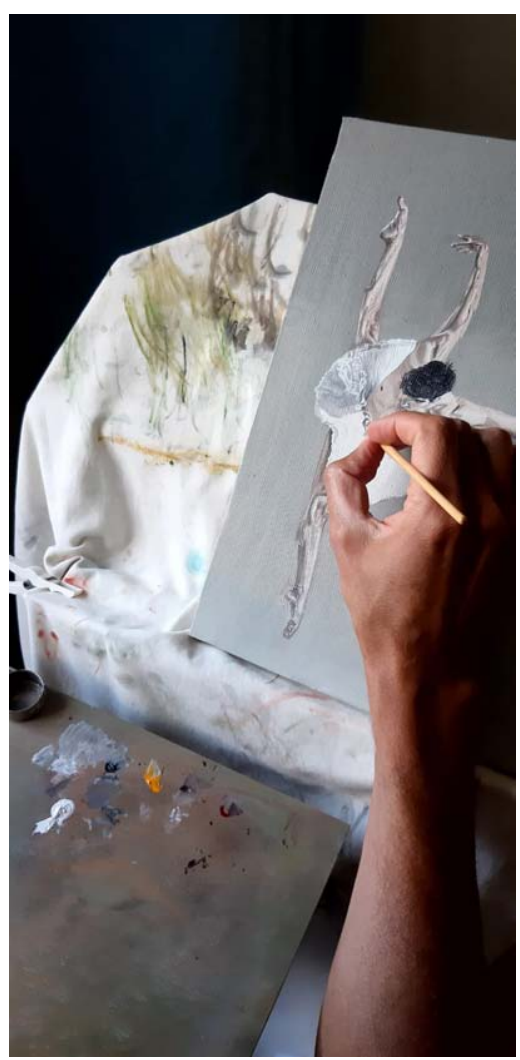

Figure 5. Auricolari per smartphone, 2020; @ Coco Sardòn 
U T S

e PRESS
Chissà se, come scrive un autore, questi oggetti rimarranno incisi nella memoria storica individuale oppure svaniranno-soffermandoci sull'immagine di un'autrice - come il respiro sulle lenti degli occhiali, una volta tolta la mascherina? D'altronde a decretare la differenza tra l'essere e il non essere è proprio il respiro, insediato in questo tempo pandemico da un nemico micidiale e invisibile, celebrato in tutti i rimandi, reali e onirici. Il movimento ritmico del diaframma è insieme stasi e movimento, fissità e rinnovamento. Il respiro cercato dalla corritrice svedese quando si inerpica sui boschi con i suoi stivali di gomma (fig.6) è parte di un respiro globale che ingloba tutti i regni della natura, umanità e oggetti compresi: è il respiro del tempo, il nostro tempo, lasciato magari a imperitura memoria.

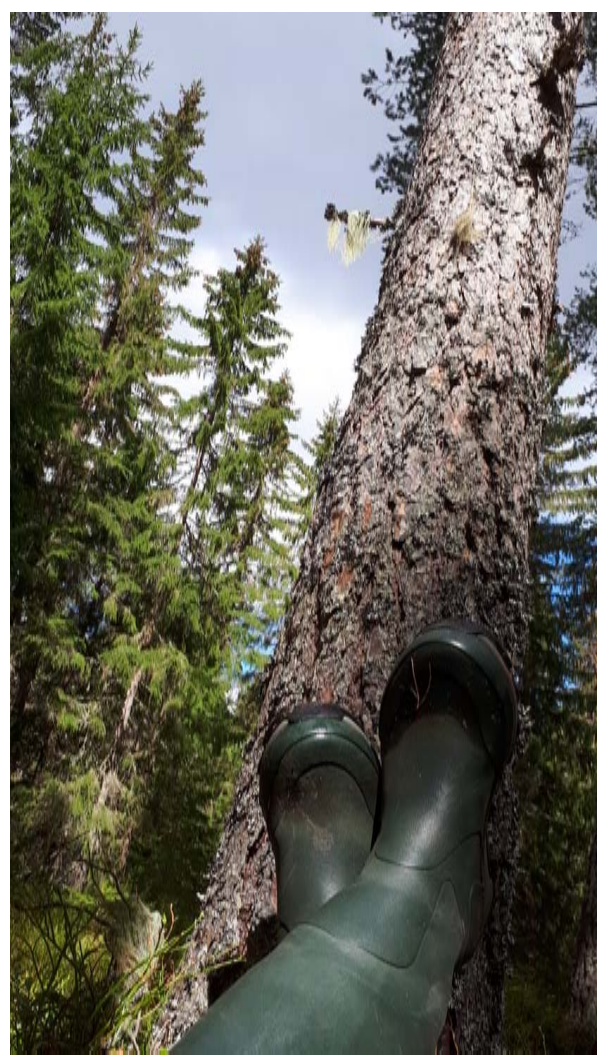

Figure 6. $\quad$ Stivali di gomma, 2020; (c) Korridor Karin

\section{Verso un nuovo presente}

In questo percorso si è voluto documentare come il patrimonio personale e d'affezione, alla pari di quello conservato in istituzioni museali, abbia la capacità di 'rispondere ad alcune domande del nostro tempo' (Amselle 2017: 31). Sono infatti sempre più diffuse esperienze di 'musei della società' (Chevallier 2013) che non si accontentano di immagazzinare ed esporre, ma che dialogano con il pubblico anche attraverso oggetti non musealizzati in grado di 'parlare di noi e del nostro esistere' (Bodo et al. 2016: 21).

D'altra parte, 'la centralità delle relazioni per la vita moderna e la centralità della cultura materiale per le relazioni' (Miller 2014: 185) è un presupposto fondamentale per comprendere l'approccio de 'Il respiro del tempo,' che nasce in un momento in cui le relazioni fra persone sono state profondamente modificate a causa del lockdown.

Alla fine della mostra, conclusasi il 15 settembre, la situazione della pandemia in Italia, dopo un'estate in cui tutto era apparentemente tornato alla normalità, si è di nuovo aggravata: per questo motivo, il 5 novembre 2020, il Governo italiano ha programmato un ulteriore lockdown per quelle 'regioni rosse,' in cui 
cioè sono presenti massima gravità e alto rischio ${ }^{5}$. Il contributo non può e non vuole essere una disamina della nuova condizione, però, da giugno in avanti, è stato tangibile il desiderio da parte delle persone di ritrovare una socialità, costituita da incontri fisici, da vivere nel tempo libero o a scuola. In conclusione, l'esperienza de "Il respiro del tempo," e di molte iniziative digitali, potrebbe essere replicabile ma dovrebbe tenere conto di quanto sia forte (e lo sarà sempre di più) la spinta ad avere relazioni in presenza e non mediate da qualsiasi tipo di device.

\section{Bibliografia}

Amselle, J. L. 2017, Il museo in scena. L'alterità culturale e la sua rappresentazione negli spazi espositivi. Meltemi, Milano.

Bodo, S., Mascheroni, S., \& Panigada, M. G. 2016, 'Un patrimonio di storie: perché e per chi?’ in Un patrimonio di storie. La narrazione nei musei, una risorsa per la cittadinanza culturale, (eds.) S. Bodo, S. Mascheroni, M. G. Panigada. Mimesis, Milano: 21-22.

Chevallier, D. (ed.) 2013, Métamorphoses des musées de société. La Documentation française, Paris.

CulturaeMirabilia 2020. Online, available: https://culturaemirabilia.blogspot.com/ [Accessed 16 November 2020].

Giraud, C. 2020, 'Il Ministero della Cultura presenta un report sulla reputazione online dei musei durante il Covid.' Online, available: https://www.artribune.com/professioni-e-professionisti/2020/07/il-ministero-della-cultura-presentaun-report-sulla-reputazione-online-dei-musei-durante-il-covid/ [Accessed 16 November 2020].

Hassan, C. 2020, 'Mostra e (infiniti) percorsi espositivi.' Online, available: https://www.respirodeltempo.it/mostra-epercorsi-espositivi/ [Accessed 16 November 2020].

Il respiro del tempo 2020. Online, available: www.respirodeltempo.it [Accessed 16 November 2020].

Lacan, J. 2019, Il seminario. Libro XVI. Da un Altro all'altro, 1968-1969. Einaudi, Torino.

Miller, D. 1991, 'The Necessity of Primitive in Modern Art' in The Myth of Primitivisms: Perspectives on Art, (ed.) S. Hiller. Routledge, London, New York: 50-71.

Miller, D. (ed.) 2005, Materiality. Duke University Press, Durham.

Miller, D. 2014, Cose che parlano di noi. Un antropologo a casa nostra, il Mulino, Bologna.

Sacco, P. L. 2017, 'Appunti per una definizione di “Welfare culturale.” Online, available: http://www. ilgiornaledellefondazioni.com/content/appunti-una-definizione-di-welfare-culturale-1 [Accessed 16 November 2020].

Sgarlato, A. 2020, Nota su oggetti, desiderio e identità. Online, available: https://www.respirodeltempo.it/nota-su-oggettidesiderio-e-identita/ [Accessed 16 November 2020].

Van Gennep, A. 2012, I riti di passaggio. 2nd ed., trans. M. L. Remotti. Bollati Boringhieri, Torino.

5 Uno dei due scriventi si trova in Piemonte, regione che, come Lombardia, Valle d'Aosta e Calabria è stata classificata come zona rossa il 5 novembre 2020 . 